\title{
Le Riou Mort, affluent du Lot, pollué par métaux lourds. III. Etude faunistique générale.
}

\author{
N. Giani1
}

Le Riou Mort, ruisseau fortement dégradé par des effluents domestiques et industriels, se caractèrise par une forte pollution organique, une grande turbidité, la présence de métaux lourds et de brusques variations du pH et de la teneur en $\mathrm{O}_{2}$ en fonction des rejets ponctuels. Cette pollution est envisagée ici d'un point de vue faunistique selon deux approches: l'étude globale de la faune et l'étude de paramètres résumant les données faunistiques (densité, nombre de taxas, indice de Boumaud et Keck).

Ces deux méthodes donnent des résultats équivalents et permettent de suivre l'évolution des conditions de milieu. La seconde est la plus simple et la plus rapide et les études faunistiques générales apparaissent superflues dans de tels cas de forte pollution. De même il suffit de considérer les Oligochètes globalement, l'information apportée par leur détermination spécifique s'avérant très faible.

The Riou Mort, a tributary of the Lot polluted by heavy metals. III General faunistic study.

The Riou Mort, a river strongly degraded by domestic and industrial effluents, is characterised by a marked organic pollution, a high turbidity, the presence of heavy metals and sudden variations of $\mathrm{pH}$ and $\mathrm{O}_{2}$ content in relation to regular discharges. This pollution is examined here from the faunistic approach by two methods: the total study of the fauna and the study of parameters that summarise the faunistic data (density, number of taxa, index of Bournaud and Keck).

The two methods give similar results and can be used to describe the changes in the environment. The second method is the simplest and quickest, and a general faunistic study appears superfluous in such cases of gross pollution. Likewise, it is sufficient to consider the total oligochaetes only because very little additional information is obtained from their specific identification.

\section{1. - Milieu et méthodes.}

Le Riou Mort draine le bassin houiller de Decazeville dans le département de l'Aveyron. Il prend sa source à $520 \mathrm{~m}$ d'altitude et, après un cours de $23 \mathrm{~km}$, il se jette dans le Lot à $185 \mathrm{~m}$ d'altitude.

\section{1. - Les stations d'étude (fig. 1)}

- Station 1 : située dans un secteur boisé, pauvrement agricole, sur un sol de grès rouges argileux du Permien, elle a été choisie comme station de référence. Distance à l'embouchure : $18 \mathrm{~km}$; altitude

1. Laboratoire d'Hydrobiologie, ERA 702 du C.N.R.S., Université Paul Sabatier, 118, route de Narbonne, 31062 Toulouse Cedex.
$261 \mathrm{~m}$; lit de $1,5 \mathrm{~m}$ de largeur constitué de seuils et de mouilles ; vitesse du courant : 0,4 à $0,8 \mathrm{~m} / \mathrm{s}$; à sec en août 1976 où il ne subsistait que quelques flaques d'eau.

- Station 2 : à l'entrée de Firmi ; distance à l'embouchure : $13 \mathrm{~km}$; altitude : $241 \mathrm{~m}$; largeur du lit : $2,5 \mathrm{~m}$; vitesse du courant : 0,6 à $0,9 \mathrm{~m} / \mathrm{s}$; polluée organiquement par les eaux d'égoûts de la ville de Firmi ; en été les eaux ont une couleur bleue et il y a un fort développement de Sphaerotilus natans.

- Station 3 : à l'entrée de Decazeville, en amont des aciéries. Distance à l'embouchure : $8 \mathrm{~km}$; altitude : $215 \mathrm{~m}$; largeur : $3 \mathrm{~m}$; vitesse du courant : 0.6 à $0,8 \mathrm{~m} / \mathrm{s}$; forte pollution organique par les eaux usées de Firmi et les premières cités de Decazeville (17 000 habitants).

- Station 4 : à la sortie de Decazeville, en amont de Viviez. Distance à l'embouchure : $5,5 \mathrm{~km}$; alti- 


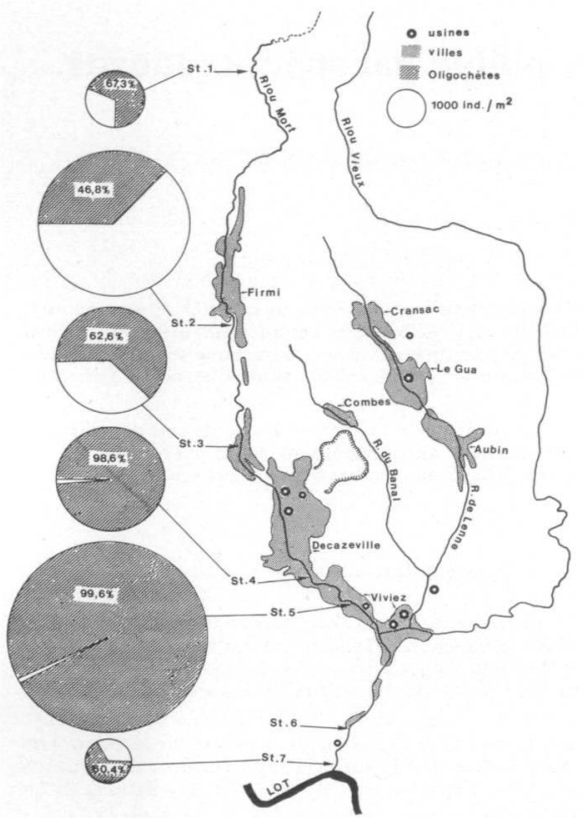

Fig. 1. Réseau hydrographique, localisation des stations d'étude et des principales sources de pollution. Les cercles indiquent la densité de peuplement et l'impor. tance des Oligochètes aux diverses stations.

tude : $205 \mathrm{~m}$; largeur : $3,5 \mathrm{~m}$; vitesse du courant : 0,3 à $0,5 \mathrm{~m} / \mathrm{s}$; forte pollution organique à laquelle s'ajoutent les eaux usées des aciéries qui confèrent au Riou Mort une couleur brun-chocolat.

- Station 5: dans Viviez (3000 habitants), en amont de la confluence avec le Riou Vieux qui est l'affluent principal du Riou Mort. Distance à l'embouchure : $4 \mathrm{~km}$; altitude : $197 \mathrm{~m}$; largeur : $3,5 \mathrm{~m}$; vitesse du courant : 0,3 à $0,6 \mathrm{~m} / \mathrm{s}$; caractéristiques identiques à la précédente.

- Station 6: en aval du Riou Vieux. Distance à l'embouchure : $600 \mathrm{~m}$; altitude : $190 \mathrm{~m}$; largeur : $5 \mathrm{~m}$; vitesse du courant : 0,5 à $0,6 \mathrm{~m} / \mathrm{s}$; le Riou Vieux reçoit, avant de se jeter dans le Riou Mort, les effluents d'une fonderie de zinc et surtout le ruis- seau de Lenne pollué par les égoûts des cités de Cransac et Aubin, par diverses usines métallurgiques et par une fabrique de peinture.

- Station 7: après la centrale thermique qui déverse dans le Riou Mort ses eaux usées pompées depuis le Lot. Distance à l'embouchure : $50 \mathrm{~m}$; altitude : $186 \mathrm{~m}$; largeur : $5 \mathrm{~m}$; vitesse du courant : $0,5 \mathrm{~m} / \mathrm{s}$.

\section{2. - Paramètres physico-chimiques}

La physico-chimie du Riou Mort a fait l'objet de plusieurs études (Say, 1978; Say et Giani, 1981). Nous soulignerons ici les principales caractéristiques du milieu, résumées de ces travaux et complétées par des données ultérieures 1 .

\subsection{1. - Température}

Les amplitudes thermiques annuelles sont de l'ordre de $14^{\circ} \mathrm{C}$ à la station 1 et atteignent $18^{\circ} \mathrm{C}$ à la station 7 (fig. 2 et $3 \mathrm{~A}$ ). La température croît progressivement de la station 1 à la station 3 puis brutalement en 4 avec l'arrivée des rejets des aciéries (ce réchauffement est plus marqué en été : 7 à $8^{\circ} \mathrm{C}$ ); elle se stabilise ou décroît légèrement après la confluence avec le Riou Vieux et remonte faiblement après la centrale thermique (st. 7).

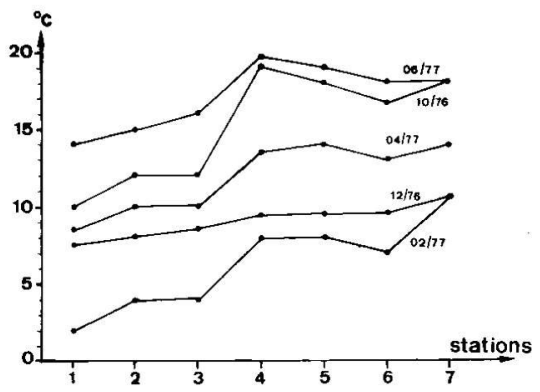

Fig. 2. Evolution spatio-temporelle de la température de l'eau.

1. Nous remercions l'Agence Financière du Bassin AdourGaronne qui a mis à notre disposition certains de ses résultats. 

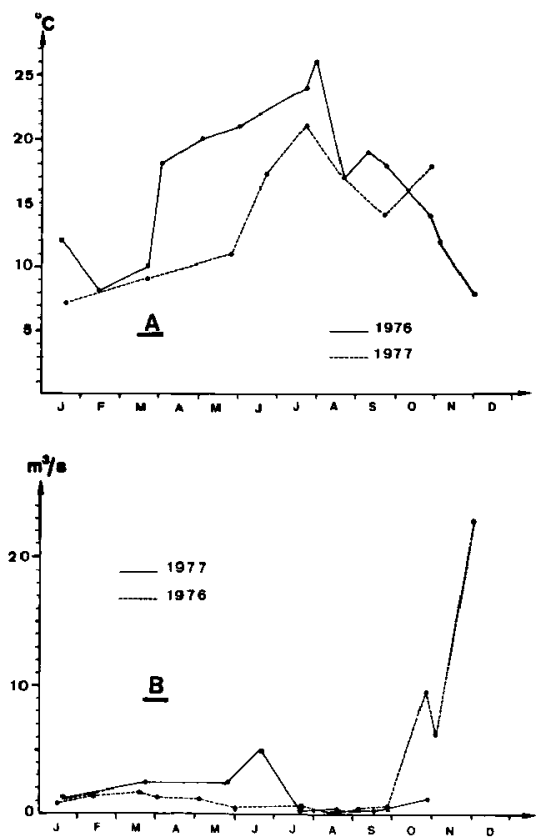

Fig. 3. Evolution annuelle de la température de l'eau (A) et du débit (B) à la station 7 .

\subsection{2. - Matières en suspension}

Elles ont été évaluées par pesée du résidu sec après filtration. A la station 1 , la quantité de matières en suspension est toujours très faible $1<$ à $25 \mathrm{mg} / \mathrm{l}$ ) et l'eau est limpide ; lors de fortes précipitations, l'érosion des grès rouges confère à l'eau une teinte rouge brique. Après l'arrivée des effluents des aciéries (st. 4) l'eau prend une couleur brun-chocolat due aux matières en suspension (jusqu'à $500 \mathrm{mg} / \mathrm{l}$ ). En aval il y a une sédimentation progressive et à l'embouchure la teneur en matière en suspension n'est plus que de 30 a $100 \mathrm{mg} / \mathrm{l}$.

Les teneurs en matière en suspension sont fortement corrèlées avec le débit comme l'illustre la figure 4. C'est en période de crue (décembre 1976)

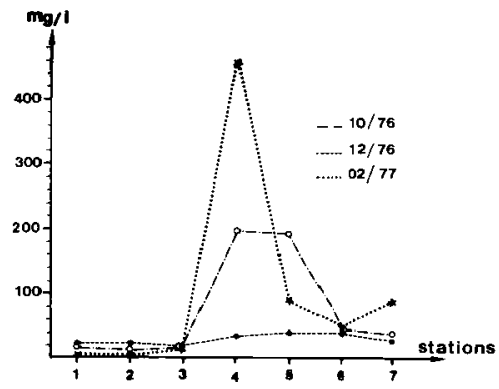

Fig. 4. Teneur en matière en suspension dans l'eau aux diverses stations ( 3 exemples mettant en évi. dence la relation particulière avec le débit).

que, malgré la forte érosion et du fait de la dilution, les teneurs sont les plus faibles. En période de basses eaux (02/77) les teneurs sont maximales mais la sédimentation est plus rapide qu'en période normale $(10 / 76)$ où les teneurs sont moindres.

\subsection{3. - Débit}

Il a été mesuré à la station $7:$ moins $\mathrm{d}^{\prime} 1 \mathrm{~m}^{3 / \mathrm{s}}$ en été, moins de $2 \mathrm{~m} 3 / \mathrm{s}$ en hiver (fig. $3 \mathrm{~B}$ ). Cependant, lors des crues les débits peuvent atteindre $23 \mathrm{~m}^{3} / \mathrm{s}$ (novembre 1976). A la station 1 le lit peut s'assécher en été ; aux autres stations, le débit d'étiage est constitué à $90 \%$ par des rejets d'eaux usées industrielles et domestiques (Sodeteg, 1976).

\subsection{4. - Le $p H$}

Légèrement basique $(7,5$ à 8,2$)$ aux trois premières stations, le pH décroît faiblement à la station 4 (7 à 7,8 ) et après la confluence avec le Riou Vieux (6.5 à 7,3). Dans la zone aval il est soumis à d'amples et brutales variations dues aux déversements intermittents des usines. Soit il augmente de plusieurs unités instantanément (de 6,5 à 9,4 selon Say, 1978), soit il diminue tout aussi brutalement : de 6,8 à 3,8 le $01 / 06 / 76$ à 10 heures.

\subsection{5. - Oxygène dissout}

Les teneurs sont toujours voisines de la saturation aux 4 premières stations, mais on note un défi- 
cit marqué au cours des mois les plus chauds aux stations 2,3 , et 4 . Les stations 5,6 et 7 se caracterisent par des variations brutales de la teneur en $\mathrm{O}_{2}$ généralement concomitantes des variations de $\mathrm{pH}$ et résultant des rejets polluants. Ainsi les taux de saturation sont généralement compris entre 70 et $120 \%$ à la station 7 (fig. $5 \mathrm{~A}$ ), mais ils peuvent chuter à $32 \%$ comme le $01 / 06 / 76$ à $10 \mathrm{~h}$ à la suite du rejet précité.

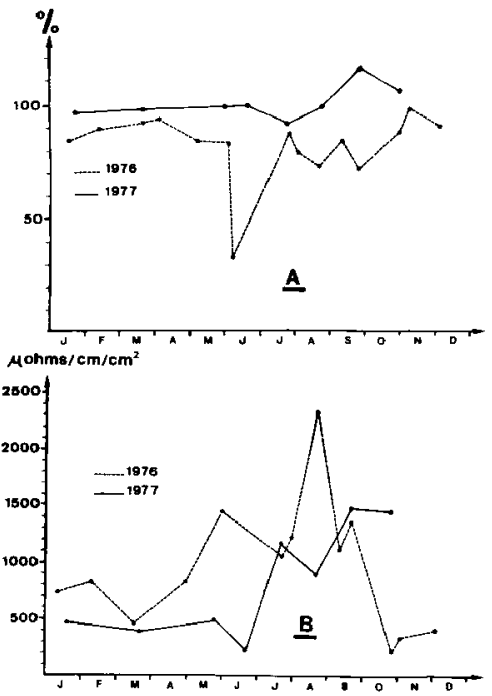

Fig. 5. Evolution annuelle du taux de saturation en $\mathbf{O}_{2}$ (A) et de la conductivité (B) à la station 7 .

\subsection{6. - Conductivité à $20^{\circ} \mathrm{C}$}

Les deux séries de mesures effectuées (tableau I) montrent que la conductivité augmente brutalement à la station 4 après les aciéries. Puis elle décroît progressivement jusqu'à la confluence avec le Lot mais elle est alors sujette à de brutales variations en fonction des déversements des usines chimiques. Ainsi, à la station 7 (fig. $5 \mathrm{~B}$ ), elle varie fortement en fonction du débit et des effluents : les valeurs observées oscillent entre 250 et $2350 \mu \mathrm{ohms} / \mathrm{cm} / \mathrm{cm}^{2}$ avec une moyenne de $907 \mu \mathrm{ohms} / \mathrm{cm} / \mathrm{cm}^{2}$.

Tableau I. Conductivités à $20^{\circ} \mathrm{C}\left(\mathrm{en} \mu \mathrm{ohms} / \mathrm{cm} / \mathrm{cm}^{2}\right)$ relevées aux diverses stations à deux périodes de l'année 1976.

\begin{tabular}{lccccccc}
\hline Stations & 1 & 2 & 3 & 4 & 5 & 6 & 7 \\
\hline Avril & 393 & 448,7 & 403,8 & 798,7 & 864 & 644,1 & 334,1 \\
Juin & 414,7 & 477,3 & 372,6 & 799,5 & 797 & 638 & 617,1 \\
\hline
\end{tabular}

\subsection{7. - Les principaux ions}

Le tableau II regroupe les valeurs moyennes relevées par Say (1978) pour chacun des principaux ions. Seule la teneur en silice est relativement constante tout le long du cours. La teneur en $\mathrm{Cl}$ augmente de la station 3 à la station 6, c'est-à-dire tout au long de la traversée des agglomérations; le $\mathrm{Cl}$ provient donc essentiellement des eaux usées des égoûts. La concentration des autres ions augmente brutalement après les rejets des usines qui constituent donc la source principale d'enrichissement en ces divers ions. L'exemple le plus remarquable est celui du $\mathrm{SO}_{4} \mathrm{~S}$ qui passe brusquement de 15,4 à $598 \mathrm{mg} / 1$ (soit 39 fois plus). Les teneurs redécroissent ensuite progressivement vers l'embouchure.

Tableau II. Concentrations moyennes (en $\mathrm{mg} / \mathrm{l}^{-1}$ ) calculées pour les principaux ions à certaines des stations d'étude.

\begin{tabular}{cccccccc}
\hline $\begin{array}{r}\text { ions } \\
\text { Stations }\end{array}$ & $\mathrm{Na}^{+}$ & $\mathrm{K}^{+}$ & $\mathrm{Mg}^{2+}$ & $\mathrm{Ca}^{2+}$ & $\mathrm{SO}_{4}^{2-}$ & $\mathrm{Si}$ & $\mathrm{CL}^{-}$ \\
\hline $\mathbf{1}$ & 12,6 & $\mathbf{8 , 4}$ & $\mathbf{1 9}$ & $\mathbf{6 0}$ & $\mathbf{1 4}$ & $\mathbf{1 0 , 5}$ & 5 \\
3 & 11,8 & $\mathbf{8 , 4}$ & 15,5 & $\mathbf{4 6}$ & 15,4 & 10,1 & 14,7 \\
5 & 35,8 & 14,3 & 93 & 64,6 & 598 & 119 & 17,3 \\
6 & 32 & 16 & 60 & 59 & 495 & 11,8 & 28 \\
7 & 14,9 & 8 & 31 & 29,5 & 148 & 9,5 & 14,2 \\
\hline
\end{tabular}

Le tableau III regroupe l'évolution annuelle des principaux ions à la station 7 . Il indique la grande variabilité des teneurs en fonction du débit, de l'importance et de la nature des divers rejets des usines.

\subsection{8. - Le substrat}

Say et Giani (1981) ont dosé les métaux lourds dans les sédiments de certaines des stations étudiées 
Tableau III. Concentrations (en $\mathrm{mg} / \mathrm{l}$ ) des principaux ions, relevées sur un cycle annuel de mesure (1976), à la station 7.

\begin{tabular}{|c|c|c|c|c|c|c|c|c|c|c|c|c|c|}
\hline ions & mois & 01 & 02 & 03 & 04 & 05 & 06 & 07 & 08 & 09 & 10 & 11 & 12 \\
\hline $\mathrm{Ca}^{2+}$ & & 47 & 96 & 52 & 42 & 60 & 120 & 115 & 180 & 120 & 28 & 38 & 24 \\
\hline $\mathbf{M g}^{2+}$ & & 45 & 36 & 32.4 & 27 & 48 & 58 & 80 & 195 & 105 & 7 & 21,6 & 12 \\
\hline $\mathrm{Na}^{+}$ & & 38 & 26 & 19,2 & 23,5 & 30 & 44 & 97,5 & 86 & 60 & 5 & 28 & 7.4 \\
\hline $\mathrm{K}^{+}$ & & 10 & 8,7 & 6,6 & 8 & 10 & 11.6 & 15.5 & 38 & 16.5 & 4,2 & 6.5 & 5,2 \\
\hline $\mathrm{NH}_{4}$ & & 2,2 & 1,2 & 1,1 & 1,2 & 2,8 & 2.9 & 1.3 & 1,4 & 2,4 & 0.8 & 0,6 & 1.4 \\
\hline $\mathrm{HCO}_{3}^{-}$ & & 116 & 128 & 116 & 140 & 140 & 165 & 110 & 177 & 190 & 79 & 90 & 67 \\
\hline $\mathrm{CL}^{-}$ & & 35 & 21,8 & 13 & 18,1 & 20,8 & 29.2 & 24,6 & 27,6 & 28 & 13,3 & 8 & 9.2 \\
\hline $\mathrm{SO}_{2}^{2}$ & & 380 & 370 & 160 & 190 & 320 & 650 & 650 & 1530 & 650 & 70.8 & 150 & 48.4 \\
\hline
\end{tabular}

dans ce travail où nous avons seulement estimé la matière organique par la méthode de la perte au feu $\left(2 \mathrm{~h}\right.$ à $\left.550^{\circ}\right)$.

\section{Perte au feu St 1 St 2 St 3 St 4 St 5 St 6 St 7 en $\%$}

des sédiments $0,92 \quad 12,04 \quad 23,94 \quad 19,62 \quad 18,68 \quad 3,8 \quad 8,16$

A la station 1, le substrat est pauvre en matière organique: il y a ensuite un enrichissement au niveau des stations situées après les rejets des égoûts (stations 2 à 5 ).

Après les aciéries (st. 4) le lit est recouvert d'une couche de vase épaisse résultant de la sédimentation des matières en suspension contenues dans les eff luents. Cette vase noire forme des bancs épais le long des berges et elle recouvre mème les pierres du milieu du lit. A chaque crue ce dépôt instable est emporté vers l'aval et les pierres du fond réapparaissent. Une grande partie de ce matériau se redépose au niveau de l'embouchure avec le Lot en une couche très épaisse et nettement stratifiée.

\section{3. - Méthodes}

Six séries de prélèvements de faune ont été effectuées, sur un cycle de un an, aux dates suivantes : $05 / 08,15 / 10$ et $13 / 12$ en $1976 ; 22 / 02,18 / 04$ et 15/06/1977. A chaque station, 5 relevés ont été réalisés lors de chaque sortie :

- 2 au filet de Surber $\left(500 \mathrm{~cm}^{2}\right.$, vide de maille : $300 \mu \mathrm{m})$ dans les zones en courant :

- 3 à l'aide de tubes en altuglass (diamètre : $8 \mathrm{~cm}$ ) dans les dépôts en courant lent.

84743 invertébrés ont été récoltés et déterminés à des niveaux variables selon les groupes taxonomiques; parmi eux il y avait 65658 Oligochètes soit $77,5 \%$ du total.

\section{2. - Approche faunistique de la pollu- tion du Riou Mort}

Le tableau IV récapitule le total des invertébrés récoltés à chacune des stations.

\section{1. - Répartition spatiale des divers taxas (fig. 6).}

- Hydra sp. : uniquement récoltées en octobre à la station $2\left(26,5\right.$ ind.$\left./ \mathrm{m}^{2}\right)$.

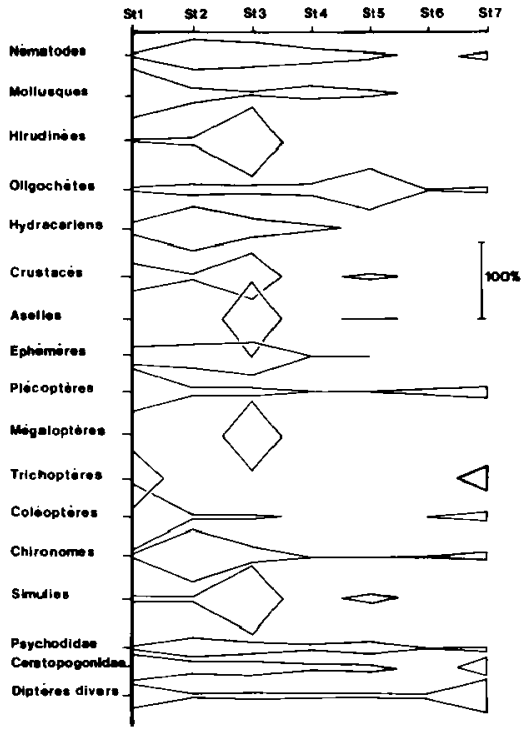

Fig. 6. Répartition longitudinale des principaux groupes d'invertébrés benthiques. 
Tableau IV. Tableau récapitulatif de la faune récoltée aux diverses stations (Coléoptères : déternninations C. Berthélemy : Ephéméroptères et Diptères autres que les Chironomides : déterminations A.G.B. Thomas).

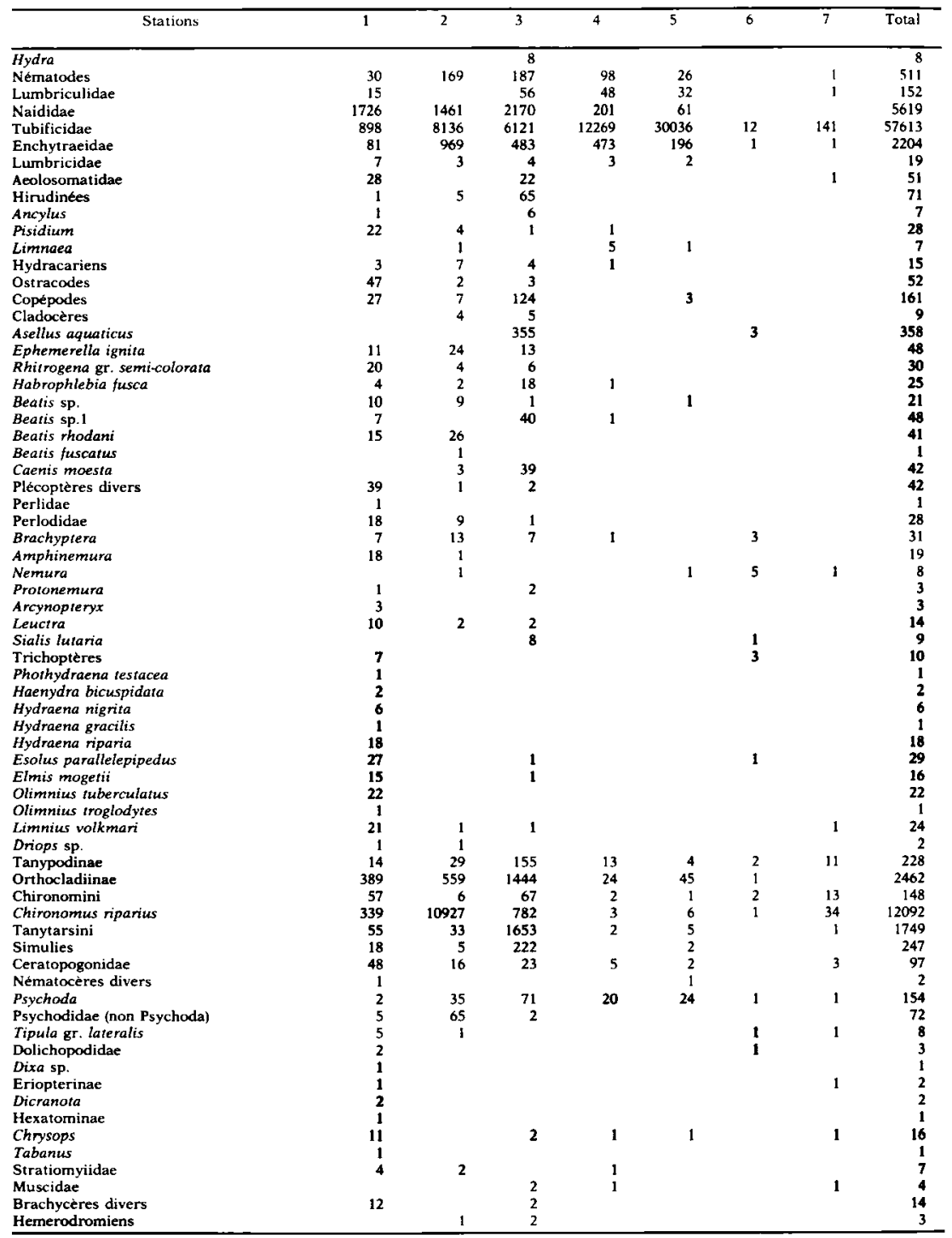


- Nématodes : absents à la station 6 , faiblement représentés en 7 , ils at teignent leur développement maximum en 2 et 3 où l'on trouve respectivement 35,2 et $32,7 \%$ du total des Nématodes récoltés. $17,8 \%$ ont été prélevés à la station 4 et seulement 5 et $7 \%$ aux stations 1 et 5 . Ils présentent une abondance maximale d'août à octobre et la plus forte densité observée est de 4428 ind. $/ \mathrm{m}^{2}$ en août à la station 4. Ce groupe contribue faiblement au peuplement de chaque station (1,3\% maximum en 3 ) mais il est, numériquement, le troisième ou quatrième groupe du benthos au niveau de certaines stations polluées organiquement $(2,3$ et 4$)$. Les Nématodes semblent par contre très sensibles aux conditions toxiques qui prévalent en 5, 6 et 7 .

- Mollusques : absents en 6 et 7 , ils constituent toujours moins de $1 \%$ du peuplement aux autres stations. $61,1 \%$ ont été récoltés en 1, $16 \%$ à chacune des stations 2 et 4 ; ce taxon est donc rare en 3 et 5 .

Les Ancylus (Oligo à $\beta$-mésosaprobes, Turoboyski 1973) sont représentés par quelques individus seu. lement au niveau des stations les moins perturbées (1 et 3). Leur présence confirme leur tolérance aux particules de charbon notée par Learner et al. (1971). Les Pisidium dominent le peuplement en Mollusques des stations 1 et 2 avec une densité maximale de 298 ind. $/ \mathrm{m}^{2}$ en octobre (st. 1); peu abondants en 3 et 4, ils disparaissent au niveau de la station 5 . Les Limnaea ( $\alpha-\beta$ mésosaprobes, Turoboyski, 1973) atteignent leur développement maximum en 4 et 5 (200 ind. $/ \mathrm{m}^{2}$ en octobre en 4 ); en 5 elles sont les seuls mollusques présents. Les divers types de Mollusques réagissent donc différemment à une pollution organique croissante - l'enrichissement en matière organique favorise les Mollusques, selon Hynes (1960) mais tous disparaissent après les agressions chimiques subies par le milieu à l'aval de Viviez.

- Hirudinées : uniquement présentes aux trois premières stations ; $90,1 \%$ ont été prélevées à la station 3 où elles n'interviennent cependant que pour $0,45 \%$ du peuplement $(0,1 \%$ en 1 et 2$)$. La densité moyenne à la station 3 est de 106 ind. $/ \mathrm{m}^{2}$; la den. sité maximum est de 270 ind $/ \mathrm{m}^{2}$ en août. Aux deux premières stations nous avons récolté Trochaeta subviridis, Dina lineata, Glossiphonia complanata et Helobdella stagnalis qui subsiste seule en 4 . Learner et al. (1971) avaient noté cette dominance d' $H$. stagnalis dans les milieux pollués riches en Oligochètes. Selon Sawyer (1974) la nourriture est le facteur dominant de la répartition des sangsues dont la réponse aux pollutions est donc indirecte. La rareté de G. complanata - considérée comme saprophile - pourrait s'expliquer par la faible abondance des Mollusques dans le Riou Mort. L'association Mollusques-sangsues a également été soulignée par Percival et Whitehead (1930).

- Oligochètes (fig. 1) : c'est le premier groupe du benthos dans le ruisseau considéré. Sa plus faible contribution numérique est de $46,8 \%$ du peuplement à la station 2 compte tenu du grand nombre de $C$. riparius présents. Les plus fortes contributions sont de $98,6 \%$ à la station 4 et de $99,6 \%$ en 5 . Aux stations 1,3 et 7 ils interviennent pour 60 à $70 \%$ des effectifs totaux. Selon les densités moyennes annuelles les stations peuvent être regroupées comme suit :

- stations 1 et 7 : densité comprise entre 2500 et 4500 ind. $/ \mathrm{m}^{2}$;

- stations 2, 3 et 4 : densité comprise entre 14500 et 22000 ind. $/ \mathrm{m}^{2}$;

- station 5 : densité de l'ordre de 77544 ind $/ \mathrm{m}^{2}$.

La répartition des récoltes d'Oligochètes reflète cette classification : $3,1 \%$ en $1,1,9 \%$ en $7 ; 14,6 \%$ en $2,10,3 \%$ en $3,15,5 \%$ en $4 ; 54,5 \%$ en 5 . Les densités maximales ont été observées en août aux stations $4\left(543272 \mathrm{ind} . / \mathrm{m}^{2}\right)$ et $5\left(525163 \mathrm{ind} . / \mathrm{m}^{2}\right)$. Des densités de 350000 ont été signalées par Richardson (1928), de 200000 par Brinkhurst (1970)...

La prépondérance des Oligochètes dans ce ruisseau tend à atténuer l'importance des autres groupes. La dominance des Oligochètes et particulièrement des Tubificidae dans les milieux pollués organiquement a été soulignée depuis fort longtemps (Richardson, 1928) et à maintes reprises depuis (Hynes, 1960 ; Brinkhurst, 1965 ; Biesiadka et Kasprzak, 1977 ...). Hynes (1957-58) considère les Oligochètes comme très sensibles aux toxiques tels le zinc. Ils tolèrent bien les sédiments riches en métaux lourds du Riou Mort comme l'ont montré Say et Giani (1981) qui ont mis en évidence des phénomènes d'accumulation chez certains d'entre eux; ils sont cependant éliminés au niveau de la station 6 .

- Hydracariens : absents en 5, 6 et 7 ; ils forment toujours moins de $1 \%$ du peuplement global. $54,7 \%$ ont été récoltés en 2 et seulement $6,2 \%$ en 4 . La densité maximale observée est de $50-60 \mathrm{ind} / \mathrm{m}^{2}$ en 3 (avril et décembre) oủ la densité moyenne annuelle est de 14 ind. $/ \mathrm{m}^{2}$.

- Crustacés (sauf Isopodes) : absents en 4, 6 et 7. $59,1 \%$ ont été récoltés à la station 3 et $31,6 \%$ en 
1. Ils constituent généralement moins de $1 \%$ du peuplement sauf à la station $1(1,8 \%)$. A cette station la densité moyenne annuelle est de 116 ind.$/ \mathrm{m}^{2}$ alors qu'elle est de 217 ind $/ \mathrm{m}^{2}$ en 3 . Les Cladocères sont très peu représentés : quelques individus en octobre et décembre aux stations 1,2 et 3 . Les Ostracodes ont un développement maximum à la station 1 (716 ind. $/ \mathrm{m}^{2}$ en août) et ils disparaissent en 4 . Les Copépodes sont présents jusqu'à la station 5 avec une abondance maximale en 3 (773 ind $/ \mathrm{m}^{2}$ en août).

- Crustacés Isopodes : 99,1\% des Aselles ont été récoltées à la station 3 où, avec seulement $2,5 \%$ du peuplement, elles constituent le $3^{*}$ groupe du benthos. La densité moyenne annuelle est de 584 ind $/ \mathrm{m}^{2}$ avec un maximum en été : $1780 \mathrm{ind} . / \mathrm{m}^{2}$ en août. L'abondance des Aselles dans les milieux pollués est bien connue (Hawkes, 1962 ; Brinkhurst, 1965, Butcher, 1957-58; Hynes, $1960 . .$.$) .$

- Ephémères (Dét. A.G.B. Thomas) : absents en 6 et 7 ; ils représentent $1,6 \%$ du peuplement en 1 et toujours moins de $1 \%$ aux autres stations. $24,5 \%$ des Ephéméroptères ont été récoltés à la station 1, $30,3 \%$ en 2 et $43,9 \%$ en 3 . Les rares captures de larves en 4 et 5 ( 3 ind.$\left./ \mathrm{m}^{2}\right)$ concernent vraisemblablement des animaux en dérive. Les densités maximales ont été observées en avril à la station 1 (646 ind. $\left./ \mathrm{m}^{2}\right)$ et en juin à la station $3\left(660 \mathrm{ind} . / \mathrm{m}^{2}\right)$. Les Ephémères sont surtout présents de décembre à août et ils ont une abondance maximale d'avril à juin. Rhitrogena gr. semi-colorata domine à la station 1 et ne se rencontre qu'en faible nombre en 2 et 3. En 2 ce sont Ephemerella ignita, Beatis sp/ et $B$. rhodani qui sont dominants ; $B$. rhodani est d'ailleurs absente dans les stations suivantes où Habrophlebia fusca et Caenis moesta présentent leur développement maximum. C. moesta a presque exclusivement été prélevée en 3. Selon Hynes (1957-58) parmi les formes que nous avons récoltées les Rhitrogena sont les plus sensibles à la pollution. Selon Turoboyski (1973) $B$. rhodani peut vivre dans des eaux $\alpha$-mésosaprobes mais elle domine dans les milieux oligasaprobes. $E$. ignita et $H$. fusca sont des espèces oligo à $\beta$-mésosaprobes selon Sladecek (1973). E. ignita est connue pour sa résistance à une certaine pollution organique (Brinkhurst, 1965 ; Thomas, 1981)

- Plécoptères : $57.1 \%$ ont été récoltés à la station $1,20,1 \%$ en $2,8,9 \%$ en 3 et $7 \%$ en 7 . Ils représentent toujours moins de $1 \%$ du peuplement sauf à la station $1\left(2,3 \%\right.$; densité moyenne : 153 ind $\left./ \mathrm{m}^{2}\right)$. La densité maximale a été observée en $2: 339$ ind. $/ \mathrm{m}^{2}$. Ce groupe considéré comme saproxène (Turoboyski, 1973) est surtout représenté pendant les mois d'hiver, d'octobre à juin. Les Perlidae et Arcynopteryx sont limites à la station 1 ; en 2 le peuplement est essentiellement formé par les Perlodidae et le genre Brachyptera qui est encore assez bien représenté en 3. Les Nemura ne sont présents qu'aux stations 5 et 7, en petit nombre. Koryak et al. (1972), qui en ont également rencontré dans des eaux acides polluées par des mines, notent l'abondance de ce genre, en Europe, dans les eaux à $\mathrm{pH}$ faible ou à forte teneur en fer. Tomkievicz et Dunson (1977) ont fait la même observation aux U.S.A.

- Trichoptères: uniquement présents aux stations 1 et 7 où ils constituent moins de $1 \%$ du peuplement. La densité maximale observée est de $\mathbf{4 0}$ ind.$/ \mathbf{m}^{2}$ en 1 .

- Mégaloptères: nous avons récolté Sialis luta. ria uniquement à la station 3 où il constitue moins de $1 \%$ du peuplement avec des densités moyennes annuelles de 14 ind. $/ \mathrm{m}^{2}$, pouvant atteindre 100 ind. $/ \mathrm{m}^{2}$ en août. La présence de cette espèce dans les milieux pollués a souvent été soulignée (Hynes 1960, Brinkhurst 1965...).

- Coléoptères (Dét. C. Berthélemy) : 86,3\% ont été récoltés à la station 1 où ils forment $2,8 \%$ du benthos (moins de $0,1 \%$ ailleurs) avec une densité moyenne de 186 ind $/ \mathrm{m}^{2}$. Sur les 12 espèces recensées 5 seulement sont bien représentées : Esolus parallelepipedus $(27,6 \%)$, Oulimnius tuberculatus $(14,6 \%)$, Limnius volkmari $(16,696)$, Hydraena riparia $(13,9 \%)$ et Elmis mogetii $(10,6 \%)$. Ce groupe considéré comme saproxène est pratiquement absent des stations polluées; seules $L$. volkmari et à un degré moindre $E$. parallelepipedus et $E$. mogetii peuvent se rencontrer aux stations 2 et 3 . Learner et al. (1971) avaient prélevé $L$. volkmari dans la rivière Cynon polluée. E. mogetii est considérée comme une forme oligosaprobe (Sladecek 1973).

- Chironomides (Dét. J.N. Tourenq) : ils représentent moins de $1 \%$ du peuplement aux stations 4 et 5 , entre 20 et $30 \%$ en 1,3 et 7 et ils sont le groupe le plus abondant du benthos en $2.70,7 \%$ des chiro nomes ont été récoltés à cette dernière station, $21 \%$ en 3 et seulement 4 et 3,5\% en 1 et 7 . L'importance des Chironomides à la station 2 résulte de la pullulation de Chironomus riparius en été : 285503 ind. $/ \mathrm{m}^{2}$ en août et 14015 ind. $/ \mathrm{m}^{2}$ en octobre. Une prolifération moindre s'observe à la station 3 : densi- 
tés allant de 3271 a 18845 ind./m² alors qu'aux autres périodes elles ne dépassent pas 500. Edwards (1955) avait noté des densités de $100000 \mathrm{ind} / \mathrm{m}^{2}$ pour cette espèce, bien connue de tels milieux, et considérée comme polysaprobe à $\alpha$-mésosaprobe (Tuboroyski 1973, Sladecek 1973). Selon Berg (1948) C. riparius serait absente en présence d'un courant fort mais Hynes (1960) l'a rencontrée en courant rapide lorsque les interstices sont colmatés par les algues, les bactéries et les particules comme dans le Riou Mort. Dratnal et Kasprzak (1980) ont observé le même phénomène.

L'abondance de $C$. riparius masque en fait la répartition des autres Chironomides qui atteignent leur abondance maximale à la station 3 et sont un element important du benthos des stations 1 et 2 . Les Tanypodinae, peu représentés en 1, ont leur abondance maximale aux stations 2 et 3 ; ceci est probablement à relier avec leur caractère * carnassier * et prédateurs d'Oligochètes comme le remarquait Hynes (1960). Les Tanytarsini sont surtout abondants à la station 3 mais celà résulte d'une prolifération saisonnière de Micropsectra atrofasciata : 1694 ind. $/ \mathrm{m}^{2}$ en aoat et 3253 en octobre ; aux autres dates ou aux autres stations les densités sont inférieures à 500 ind $/ \mathrm{m}^{2}$.

- Psychodidae : absents à la station 6, ils sont surtout abondants en 2 (44,1\% des récoltes), en 3 $(26,8 \%)$ et en $5(13,9 \%)$. Ils constituent toujours moins de $1 \%$ du peuplement et la plus forte densité moyenne s'observe en 2 (196 ind./m²). Les Psychodidae du genre Psychoda se rencontrent tout le long du cours d'eau avec deux minima en 1 et 7 et deux maxima aux stations 2 et surtout 5 . Ils tolèrent donc bien certaines des agressions, organiques ou non, dont le Riou Mort fait l'objet. Hynes (1960) note l'abondance des Psychoda dans les milieux pollués organiquement et il l'explique par leur faculté de respirer directement dans le milieu aérien. Les autres Psychodidae sont surtout représentés à la station 3 et ils sont absents en $4,5,6$ et 7 : ils tolèrent donc seulement une certaine pollution organique.

- Ceratopogonidae: ils forment généralement moins de $1 \%$ du peuplement $(1,2 \%$ en 1 et $1,4 \%$ en 7). $35,2 \%$ ont été récoltés à la station 1 (densité moyenne annuelle : 76 ind./m $\left.\mathrm{m}^{2}\right)$ et $26,3 \%$ en 7 (57 ind. $/ \mathrm{m}^{2}$ ). En 2 et 3 (14 et 17,6\% des captures) les densités ne sont plus que de $30 \mathrm{a} 40 \mathrm{ind} . / \mathrm{m}^{2}$ et elles tombent a 8 et 5 ind $/ \mathrm{m}^{2}$ au niveau des stations 4 et 5 . Les Ceratopogonidae sont parmi les groupes dont l'évolution spatiale reflète le mieux l'accentuation de la pollution vers l'aval sur le Riou Mort.

- Diptères divers : ils représentent moins de $1 \%$ du peuplement sauf aux stations $1(1 \%)$ et $7(2,2 \%)$. $33,4 \%$ ont été capturés en 1 et $46,9 \%$ en 7 . Les densités moyennes annuelles maximales ne dépassent pas 64 ind. $/ \mathrm{m}^{2}$ en 1 et 90 ind. $/ \mathrm{m}^{2}$ en 7 . Sur les 15 taxa considérés, 5 sont limités à la station 1 et seuls les Chrysops ont une certaine importance numérique et sont présents tout au long du cours.

\section{2. - Caractéristiques faunistiques des diverses stations}

Station 1 : Choisie comme station de référence, elle présente un peuplement équilibré et diversifié où les Plécoptères, Ephémères et Trichoptères sont bien représentés contrairement aux Nématodes, Hirudinées, Oligochètes et Psychodidae. Les Pisidium, Ostracodes, Amphinemura, Leuctra et Rhitrogena $\mathrm{gr}$. semi-colorata atteignent leur abondance maximale en 1. Selon Say (1978) les communautés algales à cette station sont également riches et diversifiées.

Notons l'absence des Turbellariés et le faible nombre de Trichoptères dans ce petit ruisseau où dominent les substrats sableux.

Station 2 : elle est caractérisée par la disparition (Trichoptères) ou la régression des groupes réputés saproxènes : Plécoptères, Coléoptères, Ceratopogonidae, Pisidium et Ancylus. Au contraire, les Nématodes, Hydracariens, Chironomides, Oligochètes et Psychodidae (non Psychoda) se développent. $C$. riparius, Brachyptera et $B$. rhodani atteignent leur abondance maximale à cette station où, selon Say (1978), abondent Sphaerotilus natans, Nitzschia palea, Gomphonema parvulum et Stigeoclonium tenue. $N$. palea et $S$. natans sont des formes méso à polysaprobes dont Turoboyski (1973) souligne la prédilection pour les eaux polluées par un mélange d'effluents domestiques et industriels. Butcher (1957-58) associe $G$. parvulum à ces deux espèces.

Il convient de distinguer deux communautés selon les saisons :

- l'une de décembre à juin, assez diversifièe, avec des Ephémères (E. ignita, B. rhodani), des Plécoptères (Perlodidae), des Simulies et des Coleoptères ( $L$. volkmari); $C$. riparius est absent ou faiblement représenté. 
- l'autre en été, franchement polysaprobe, où les groupes précédents sont totalement absents. Le peuplement alors dominé par $C$. riparius et les Oligochètes correspond à l'association ChironomusTubificidae-Sphaerotilus signalée par Butcher (1957-58) à laquelle s'adjoignent les Hirudinées. A cette station l'eau est raisonnablement oxygénée, compte tenu de la turbulence, et les Chironomus s'associent aux Oligochètes comme l'a signalé Hynes (1960). Tous profitent de leur faculté de capter l'oxygène à de faibles teneurs, de l'absence de prédation et de l'abondance de nourriture. Le pic d'abondance maximale des Chironomus à cette station correspond au minimum des Oligochètes ce qui traduit la compétition, déjà notée par Brinkhurst et Kennedy (1965), entre ces deux groupes. Selon ces auteurs les Chironomus rendent l'accès à l'interface vaseleau, pour respirer, difficile pour les vers qui meurent ou émigrent. Au mois d'août le lit apparaît comme recouvert de plaques rouges qui sont des agrégats de $C$. riparius.

Station 3 : son peuplement est assez semblable à celui de la précédente. Il en diffère essentiellement par la présence en été d'H. stagnalis, A. aquaticus, $S$. lutaria et de Simulies et la plus grande abondance d'Ephémères en hiver. Les taxa atteignant leur abondance maximale sont les Nématodes, $H$. stagnalis, $A$. aquaticus, $H$. fusca, $C$. moesta, E. ignita, S. lutaria, les Tanypodinae, Orthocladinae, Tanytarsini, Simulies et Psychoda qui sont pour la plupart des formes mésosaprobes. Selon Sawyer (1976), H. stagnalis domine dans les milieux pollués - comme ici - car elle se nourrit de Tubificidae, Chironomus et Aselles alors que Hynes (1960) ne la considère qu'occasionnelle dans de telles situations. Cette station correspond à la zone Asellus-Mollusques-G. parvulum-S. tenue-N. palea définie par Butcher (1957-58) où à celle que Hynes (1960) caractérise par le déclin des Chironomus, la progression des Mollusques (Limnaea), des sangsues et de S. lutaria. Les Oligochètes sont toujours abondants dans cette zone où Hynes (1960) signale en abondance des Tanytarsus. Dratnal et Kasprzak (1980) ont observé le remplacement de $C$. riparius par Micropsectra gr. praecox plus en aval ; ici ce remplacement s'effectue par M. atrofasciata dont Verneaux (1968) pensait qu'elle présentait une affinité pour les eaux pures et fraîches.

Station 4 : elle se caractérise par la très forte diminution de la diversité spécifique résultant :
- des nouveaix rejets domestiques et industriels ;

- du dépót des particules en suspension qui comble les interstices du substrat et réduit le nombre d'habitat (De March, 1976) ;

- de l'effet toxique, signalé par Koryak et al. (19. 72), dans cette zone de neutralisation où il y a précipitation du fer.

La situation est de poly à antisaprobe et il n'y a pas d'amélioration saisonnière permettant une recolonisation temporaire. Le peuplement est très fortement dominé par les Oligochètes.

Station 5 : la situation est identique à la précédente mais la communauté est encore plus réduite, pratiquement aux seuls Oligochètes et Psychoda.

Station 6 : les nouveaux rejets industriels toxiques et provoquant de brutales variations du $\mathrm{pH}$ et de la teneur en $\mathrm{O}_{2}$ entraînent la disparition totale de la faune. Les seuls invertébrés récoltés épisodiquement sont des individus en dérive; nous n'avons jamais capturé à cette station de formes à faible comportement de dérive telies les Oligochètes.

Station 7 : il y a une recolonisation partielle qui s'effectue probablement en partie depuis le Lot. Le peuplement est très fluctuant dans le temps.

\section{3. - Approches mathématiques de la pollution}

\section{1. - Nombre de taxa présents à chaque station}

L'évolution spatiale du nombre total de taxa récoltés (fig. $7 \mathrm{~A}$ ) traduit bien les diverses altérations

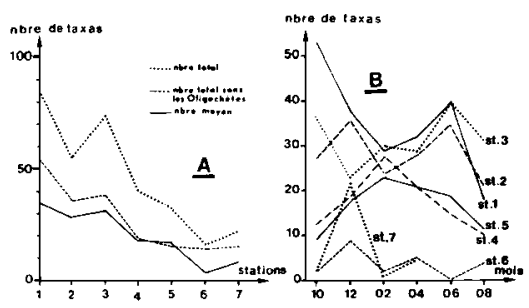

Fig. 7 Evolution du nombre de taxa : A : évolution spatiale aux diverses stations ; $\mathbf{B}$ : évolution annuelle à chaque station. 
du milieu ; maximurn en 1, ce nombre diminue après chacune des agressions : stations 2,4 et 6 . Il réaugmente en 3 et 7 ce qui confirme la récupération partielle du cours d'eau à ces locali tés. L'évolution spatiale du nombre de taxa moyen (fig. $7 \mathrm{~A}$ ) - qui intègre les fluctuations saisonnières - est identique à la précédente mais les variations sont plus atténuées.

L'évolution dans le temps du nombre de taxa présents aux diverses stations (fig. $7 \mathrm{~B}$ ) mont re que :

- les variations sont amples au niveau des stations 1,2 et 3 compte tenu de la présence saisonnière de certains taxa résultant soit du cycle biologique soit de l'amélioration périodique des conditions de milieu (hautes eaux hivernales) permettant la recolonisation:

- les variations sont faibles en 4 et 5 pour les raisons inverses des précédentes;

- les grandes variations en 7 reflètent surtout l'instabilité de ce peuplement de recolonisation.

Afin de mettre en évidence l'apport des Oligochètes dans de telles études il nous a paru intéressant de les regrouper tous en un seul taxon et de comparer la nouvelle évolution du paramètre considéré à la précédente. Cette comparaison (fig. 7 A) montre que la perte d'information est nulle à la station 6 où les Oligochètes sont absents mais qu'elle est importante en 3, 5 et 7 et ce pour des raisons différentes : en 3 et 7 le peuplement en Oligochètes se diversifie compte tenu de l'amélioration des conditions du milieu alors qu'il devient très homogène en 5. En outre la distinction entre les stations 4 et 5 bien différentes comme nous l'avons déjà vu devient difficile sans la connaissance des peuplements en Oligochètes.

\section{2. - La densité du peuplement}

La densité globale annuelle du peuplement à chaque station ( $f$ ig. $8 \mathrm{C}$ ) évolue en sens inverse du nombre de taxa ; une altération du milieu provoque une augmentation de la densité (st. 2 et 5) sauf dans les cas extrêmes avec présence de toxiques (st. 6). En 7 la densité est inférieure à celle de 1 , ce qui traduit encore une situation très altérée. La densité permet donc de séparer les diverses stations selon leur degré de pollution mais les densités en 3 et 4 sont identiques alors que la composition spécifique et le nombre de taxa présents dans ces deux stations sont très différents.
Si l"on considère la densité - à l'exclusion des Oligochètes dont la dominance influence très fortement ce paramètre - celle-ci a une évolution spatiale très différente (fig. $8 \mathrm{C}$ ) qui permet de distinguer les pollutions eutrophisantes (la densité augmente en 2) des autres (diminution en 3,4,5et 6). La densité est donc un paramètre important mais d'emploi délicat surtout si l'on considère une seule série de prélèvements, ce qui privilégie les conditions saisonnières (cas de la station 2, avec la pullulation estivale de C. riparius).

\section{3. - Autres paramètres}

La pollution du Riou Mort est très marquée et sa détection ne nécessite pas l'utilisation d'indices. Cependant pour visualiser les informations apportées par les études faunistiques précédentes nous avons calculé les paramètres proposés par Bournaud et Keck (1980) : cote moyenne d'abondance $(\overline{\mathbf{x}})$ et moment de $3^{\text {e }}$ ordre $\left(\mathbf{M}_{3}\right)$ des classes d'abondance qui s'établissent selon les formules suivantes :

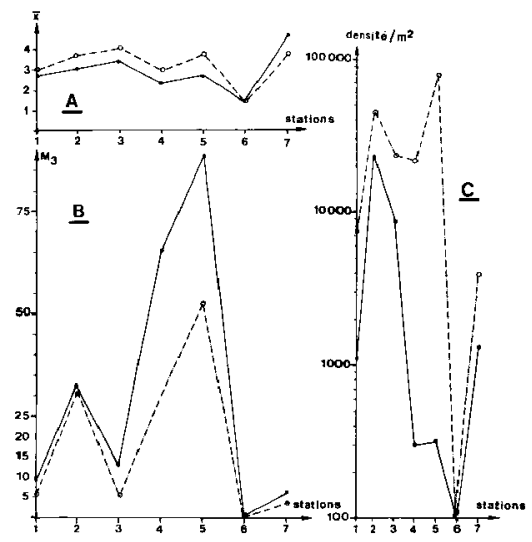

Fig. 8. Evolution de quelques paramètres du peuplement aux diverses stations : $A$ : cote moyenne d'abondance $(\overline{\mathrm{x}}) ; \mathrm{B}$ : moment d'ordre $3\left(\mathrm{M}_{3}\right)$ des classes d'abondance : $\mathrm{C}$ : densité de peuplement. Les courbes en trait plein correspondent aux valeurs des paramètres lorsque les Oligochètes sont regroupés en un seul taxon (8 A et B) ou sont exclus (8 C). 

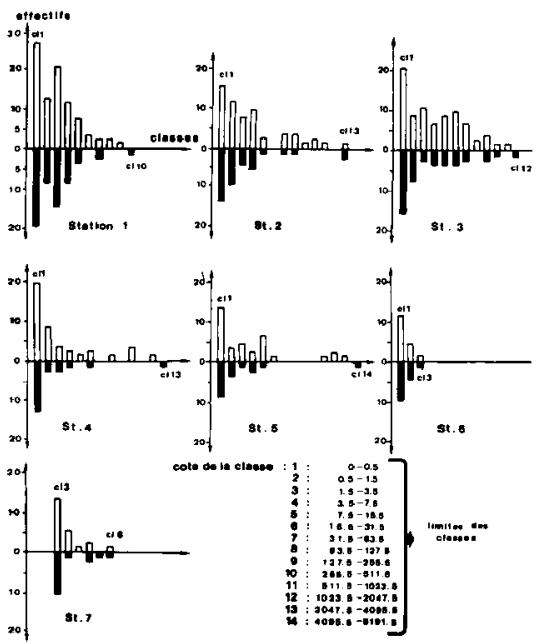

Fig. 9. Histogrammes des distributions abondance-especes aux diverses stations. Dans l'histogramme noir (sous l'axe) les Oligochètes ont été regroupés en un seul taxon.

$$
\begin{aligned}
& \overline{\mathbf{x}}=\frac{\mathbf{S x} \mathbf{X}}{\mathrm{S}} \\
& \mathbf{M}_{3}=\frac{(\mathrm{x}-\overline{\bar{x}})^{3}}{\mathrm{~S}}
\end{aligned}
$$

avec : $\mathbf{x}$, cote de la classe (voir fig. 9); $\mathbf{S x}$, nombre de taxa dans la classe de cote $x$; $S$, nombre total de taxa.

Nous avons choisi ces paramètres pour leur simplicité d'utilisation compte tenu de la forte information qu'ils détiennent : ils font intervenir l'homogénéité et la spécialisation des communautés, la diversité et la dominance (Bournaud et Keck 1980, Viaud et Lavandier 1981).

\subsection{1. - Distribution espèce-abondance}

A la station 1 les taxa à effectif faible (classes 1 et 2) et moyens (classes 3 à 5) sont prépondérants (le peuplement est diversifié) ; en 2 ils régressent et l'histogramme s'allonge vers la droite sous l'influence de certains groupes dominants (classe $\geqslant 6$ ). En 3, la distribution se rapproche de celle de 1, l'histogramme se resserre. La station 4 est caractérisée par l'effondrement des taxa moyens; ceux à faible effectif dominent mais l'histogramme s'allonge vers la droite compte tenu de l'importance des classes 11,12 et 13 ; cette importance s'accentue en 5 . En 6 , où le peuplement est accidentel, on ne rencontre que des taxa à effectif faible. En 7, le peuplement de recolonisation est constitué uniquement par des groupes à effectif moyen. La distribution especeabondance résume donc une grande partie de nos observations précédentes.

Si nous regroupons tous les Oligochètes en une seule unité systématique (fig. 9), cela provoque un regroupement et un décalage à droite des taxons dominants à la station 1 où les Oligochètes étaient donc dominants ; en 2 le phénomène est identique mais il s'accompagne d'une régression des taxons moyens. Cette modification se retrouve en $3,4,5$ et 7 (6 est un cas particulier car il n'y a pas d'Oligochètes). La détermination spécifique des Oligochètes n'apporte donc rien d'essentiel à l'information fournie par les distributions espèce-abondance.

\subsection{2. - La cote moyenne d'abondance (fig. $8 \mathrm{~A}$ )}

Selon Bournaud et Keck (1980) ce paramètre, voisin de l'indice de diversité, est très influencé par les effectifs moyens. Aux stations 2,3 et 5, les faibles variations de ce paramètre peuvent être mises en relation avec la diminution des taxa moyens. La station 4 fait exception de par la rareté des groupes à effectif moyen (classes 3 à 5).

$\mathrm{Si}$, comme précédemment, nous regroupons les Oligochètes les modifications sont très faibles et n'altèrent en rien l'allure générale de la courbe des variations spatiales de $\overline{\mathbf{x}}$; la détermination des Oligochètes n'ajoute rien à l'information contenue par ce paramètre.

\subsection{3. - Le moment d'ordre 3 des distributions des cotes dabondance (fig. $8 \mathrm{~B}$ )}

Cet indice de dominance $\left(\mathrm{M}_{3}\right)$ reflète bien l'évolution des peuplements et partant la qualité des eaux du cours d'eau étudié. Il croît brutalement à la station 2 pour décroître en 3 ce qui reflète l'améliora- 
tion des conditions de milieu. Sa forte augmentation en 4 et 5 résulte du peuplement hétérogène de ces stations fortement polluées. Sa diminution brutale en 6 ne traduit cependant pas une amélioration de la qualité des eaux car cette station est pratiquement azoïque.

Le fait de considérer globalement les Oligochètes ne change pas l'allure générale de l'évolution spatiale de $\mathbf{M}_{3}$. Au niveau des stations où la dominance des Oligochètes est moindre $(1,2,6$ et 7$)$ la modification de $\mathbf{M}_{3}$ est insignifiante. Au niveau des autres stations le fait de ne pas déterminer les Oligochètes amplifie les variations de ce paramètre; ceci peut ètre souhaitable si l'on considère que le but de tels indices est de visualiser des phénomènes difficilement perceptibles, ce qui n'est pas le cas dans notre étude.

\section{4. - Discussion}

\section{1. - Les problèmes salsonniers}

En été, le débit est constitué à 95\% par des eaux d'égoûts ; ceci, allié à la température, fait que l'été est la période la plus critique au niveau des stations polluées organiquement (2 et 3 ) : le substrat est modifié par l'abondante couverture de $S$. natans et les $C$. riparius, $A$. aquaticus, $S$. lutaria... atteignent leur développernent maximum. Dans ces mêmes stations, en hiver, on rencontre un peuplement relativement diversifié avec des Plécoptères et des Ephémères. L'évolution de $\mathbf{M}_{3}$ à chaque station montre que ce paramètre passe par un maximum en été et un minimum en hiver. Il faut faire une exception pour la station 3 (la moins polluée de celles qui le sont) où le changement complet de faune entre l'hiver et l'été maintient la diversité spécifique et $\mathbf{M}_{3}$ à un niveau raisonnable. De tels paramètres ne doivent pas être employés seuls et toujours avec prudence.

La station 4 illustre bien les variations saisonnières : 5 taxa représentés en juin et août, 17 en hiver et au printemps. De ce point de vue les Oligochètes apparaissent comme les plus stables dans le temps et donc les moins assujettis aux conditions passagères de milieu.

\section{2. - Les problèmes de blotopes}

Nos échantillonnages en courant lent (tubes) et en courant rapide (filet de surber) nous permettent de distinguer trois types de taxa :

- ceux que nous avons récoltés préférentiellement en courant : Hirudinées, Ancylus, Hydracariens, Aselles, Coléoptères, Ephémères, Plécoptères, Simulies ;

- ceux prélevés surtout en courant faible ou nul : Nématodes, Oligochètes, Limnées, Pisidium, Ostracodes, Chironomini, Pyschodidae, Sialis... ;

- ceux, plus rares, rencontrés indifféremment dans l'un ou l'autre des biotopes: Ceratopogonidae, Tanytarsini et Orthocladinae. Ceci souligne, une fois de plus, la nécessité de couvrir tous les biotopes pour avoir une idée, la plus exacte possible, du peuplement d'une station.

A la station 1, les Oligochètes sont surtout abondants en faciès lénitique. Ceci est également vrai pour les autres stations où les modifications du substrat avec la pollution ( $S$. natans, colmatage) et l'élimination des concurrents ou prédateurs permet l'augmentation de la densité des Oligochètes indifféremment dans les deux faciès. En milieu lotique les densités sont alors équivalentes ou supérieures à celles observées en faciès lénitique dans les stations non polluées comme l'avait noté Lafont (1977).

Le Riou Mort est sujet à des crues qui nettoient le lit des $S$. natans ou de la vase qui recouvre les pierres à l'aval des usines de Decazeville. Ces sédiments sont emportés, avec leur peuplement, vers le Lot et ils se redéposent abondamment à l'embouchure. Zieba et Zacwilichowska (1966) ont observé que, dans la Vistule polluée, les crues hivernales détruisent le peuplement en emportant les sédiments. Dans le Riou Mort, les stations concernées sont surtout peuplées d'Oligochètes qui recolonisent rapidement le milieu à partir des berges moins érodées et des pierres qui constituent un abri lors des crues ; après la crue de décembre 1976, il suffisait de soulever une pierre pour constater qu'elle abritait de nombreux Oligochètes alors qu'à côté le peuplement était quasiment nul.

\section{3. - Remarques sur l'intérêt des Oligochètes}

Compte tenu des grandes variations des parametres physico-chimiques (notamment $\mathrm{pH}$ et $\mathrm{O}_{2}$ ) la 
connaissance de milieux tels le Riou Mort nécessite souvent un enregistrement permanent. L'étude des communautés benthiques donne généralement une image correcte de la qualité des eaux. Une telle étude peut être réalisée globalement, comme ici et comme celà a été fait maintes fois sur de tels cours d'eau fortement pollués. Il nous apparaît clairement que les résultats sont toujours concordants et que l'essentiel a été écrit depuis fort longtemps (Richardson 1928, Hynes 1960). La mesure de quelques paramètres simples (densité, distribution espèceabondance, $\overline{\mathbf{x}}, \mathrm{M}_{3}$...) nous semble suffisante à condition de prendre en compte les variations saisonnières et les différents biotopes. Mieux vaut réserver les études complètes à des milieux dont l'altération n'est pas perceptible au premier abord ou étudier en détail un groupe donné. Quel groupe ? Il est évident que comme l'ont souligné de nombreux auteurs (Hynes 1960, Bournaud et Keck 1980, Thomas 1981) les Plécoptères et les Ephéméroptères apportent beaucoup d'information ; leur intérêt, associés aux Triclades, Coléoptères, etc... est évident dans les cas de pollution faible. Mais, trop sensibles ils disparaissent rapidement en présence d'une pollution plus forte. Les Oligochètes comme l'ont souligné Bournaud et Keck (1980) et le présent travail ont une évolution inverse. Mais compte tenu de leur faible attrait et de leur petite taille, ils sont souvent délaissés par les hydrobiologistes. Certes, comme nous l'avons montré, toute étude générale peut se dispenser de leur détermination spécifique : ils apportent peu à l'information détenue par certains paramètres $\left(\vec{x}, M_{3}\right)$ ou ils inversent le sens de l'évolution de certains autres (densité). Certains Oligochètes semblent très tolérants vis à vis de nombreuses pollutions si l'on en juge par les densités de leurs peuplements. Le cycle biologique de nombreux arthropodes leur permet de s'accommoder de certaines améliorations temporaires (saisonnières surtout) du milieu alors que la stabilité dans le temps des peuplements d'Oligochètes semble plus apte à refléter les conditions les plus défavorables du milieu. L'étude des seuls Oligochètes apporterait-elle autant d'information sur un milieu pollué qu'une étude globale ? Seraitelle susceptible de lever cette contrainte pour les hydrobiologistes d'étudier une station à plusieurs périodes de l'année ? Dans un prochain travail, nous tenterons de répondre à ces questions.

\section{Travaux cites}

Berg (K.). 1948. - Biological studies on the river Susaa. Folia limnol. Scand., $4: 1.318$.

Biesiadka (E.) et Kasprzak (K.). 1977. - An investigation on the macrofauna of the river Warta within the city of Poznan. Acta Hydrobiol. $19: 109-122$

Bournaud (M.) et Keck (G.). 1980. - Diversité spécifique et structure des peuplements de macro-invertébrés benthiques au long d'un cours d'eau : le Furans (Ain). Acta Oecologia, Oecol. Gener., $1: 131-150$.

Brinkhurst (R.O.). 1965, - Observations on the recovery of a British river from gross organic pollution. Hydrobiologia, 25 : 9-51.

Brinkhurst (R.0.). 1970, - Distribution and abundance of tubificid (Oligochaeta) species in Toronto Harbour, Lake Ontario. J. Fish. Res. Bd. Canada, 27 : $1961-1969$.

Brinkhurst (R.O.) et Kennedy (C.R.). 1965. - Studies on the biology of the Tubificidae in a polluted stream. J. Anim. Ecol, $34: 429-443$.

Butcher (R.W.). 1957.58. - Biological assessment of river pollution. Proc. Linn. Soc. of London, $170: 159.165$.

De March (B.G.E.). 1976. - Spatial and temporal patterns in macro benthic stream diversity. J. Fish. Res. Bd. Canada, 33 ; 1261 1270 .

Dratnal (E.) et Kasprzak (K.). 1980. - The response of the invertebrate fauna to organic pollution in a well oxygenated karst stream exemplified by the Pradnik Stream (South Poland). Acta Hydrobiol., 22 : 263.278.

Edwards (R.W.). 1955. - Annual Report Freshwater Biological Association, $23: 37-38$.

Hawkes (H.A.). 1962. - Biological aspects of river pollution. Pages 311432. In L. Klein, River pollution, Butterworth, London, $456 \mathrm{p}$.

Hynes (H.B.N.). 1957-58. - The use of invertebrates as indicators of river pollution. Proc. Linn. Soc. of London, 170 : 165-169.

Hynes (H.B.N.). 1960, - The biology of polluted waters. Liverpool University Press, Liverpool, 202 p.

Koryak (M.), Shapiro (A.) et Sykora (L.). 1972. - Riffle zoobenthos in st reams receiving acid mine drainage. Water Research, 6 : 1239.1247.

Lafont (M.). 1977. - Les Oligochètes d'un cours d'eau montagnard pollue : le Bief Rouge. Annls Limnol, 13: 157-167.

Learner (M.A.), Williams (R.), Harcup (M.) et Hughes (B.D.) 1971. A survey of the macrofauna of the river Cynon, a polluted tributary of the River Taff (South Wales). Freshw. Biol., 1: 339-368.

Percival (E.) et Whitehead (H.). 1930. - Biological survey of the River Wharfe. J. Ecol, $18 ; 285-302$.

Richardson (R.E.). 1928. - The bottom fauna of the middle Illinois River, 1913-1925. Bull. III. Lab. Nat. Hist., $17: 387-475$.

Sawyer (R.T.). 1974. - Leeches (Annelida : Hirudinea) in Pollution Ecology of Freshwater Invertebrates, Hart (C.W.J.) et Fuller (S.L.H.) éditeurs, Academic Press, New York and London, $389 \mathrm{p}$

Say (P.J.). 1978. - Le Riou Mort, affluent du Lot pollué par métaux lourds. I. Etude préliminaire de la chimie et des algues benthiques. Annls Limnot., $14: 113-131$.

Say (P.J.) et Giani (N.). 1981. - The Riou Mort, a tributary to the river Lot polluted by heavy metals. II. Accumulation of zinc by Oligochaetes and Chironomids. Acta Oecologia, Oecol. Applic. 2 : 339-355.

Sladecek (V.). 1973. - System of water quality from the biological point of view. Arch. Hydrobiol., $7: 1-218$.

S.O.D.E.T.E.G. 1976. - Présentation et synthèse des études menées sur la vallée du Lot dans le cadre de l'operation * Lot, rivière claire *. Toulouse, $247 \mathrm{p}$. 
Thomas (A.G.B.). 1981. - Travaux sur la taxonomie, la biologie et l'écologie d'insectes torrenticoles du Sud-Ouest de la France (Epheméroptères et Diptères : Dixidae, Cecidomyiidae, Rhagionidae et Athericidae) avec quelques exemples de perturbations par l'Hornme. These Doctorat. Toulouse, $330 \mathrm{p}$.

Tomkiewicz (S.M.) et Dunson (W. A.) 1977. - Aquatic insect diversity and biomass in a stream marginally polluted by acid strip mine drainage. Wot. Res. $11: 397.402$.

Turoboyski (L.). 1973. - The indicators organims and their ecological variability. Acto Hydrobiol., Krakow, 15 ; 259.274.
Vemeaux (J.) 1968. - Contribution à l'étude des Chironomides (Diptères, Nématocères) de la région de Besse en Chandesse (Puy-de-Dòme). Annis Sim. Limnol. Besse, 3: 119.146.

Viaud (M.) et Lavandier (P.). 1981. - Comparaison de quelques indices usuels d'évaluation de la qualité des eaux appliqués a la détection de pollutions faibles. Bull. Soc. Hist. Nat; Toulouse, $117: 221$-230.

Zieba (J.) et Zacwilichowscha (K.). 1966. - Fauna denna Wisly pomiedzy Oswiecimiem a Krakowem. Acta Hydrobiol., 8 (suppl. 1) : $389-410$. 